Document downloaded from:

http://hdl.handle.net/10251/67541

This paper must be cited as:

Martínez Tornell, S.; Hernández Orallo, E.; Cano Escribá, JC.; Tavares De Araujo Cesariny Calafate, CM.; Manzoni, P. (2013). An analytical evaluation of a Map-based Sensor-data Delivery Protocol for VANETs. 14th IEEE International Symposium on a World of Wireless, Mobile and Multimedia Networks (WoWMoM 2013). IEEE.

doi:10.1109/WoWMoM.2013.6583405.

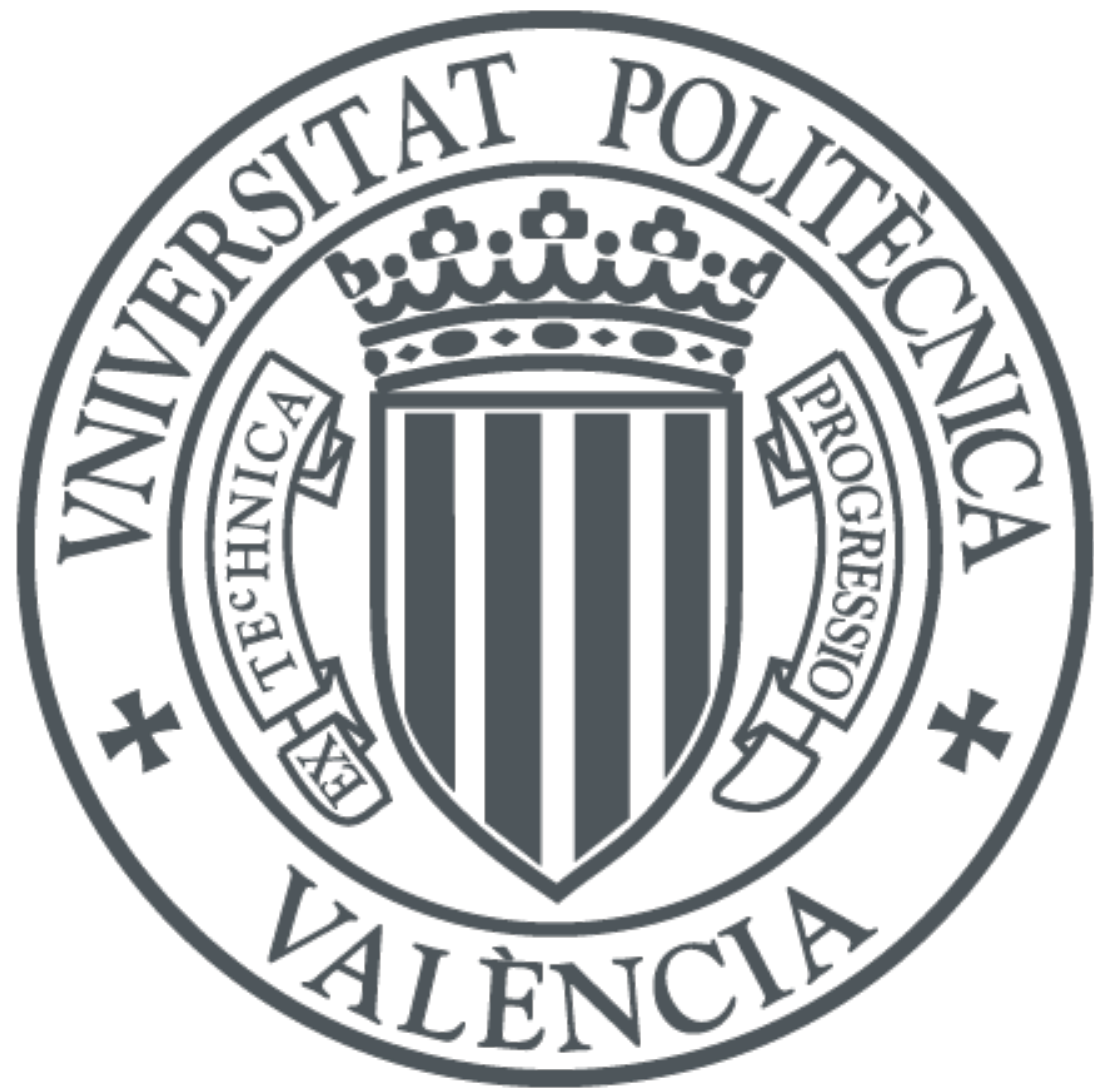

The final publication is available at

http://dx.doi.org/10.1109/WoWMoM.2013.6583405

Copyright IEEE

Additional Information

(C) 2013 IEEE. Personal use of this material is permitted. Permission from IEEE must be obtained for all other uses, in any current or future media, including reprinting/republishing this material for advertising or promotional purposes, creating new collective works, for resale or redistribution to servers or lists, or reuse of any copyrighted component of this work in other works. 


\title{
An Analytical Evaluation of a Map-based Sensor-data Delivery Protocol for VANETs
}

\author{
Sergio Martínez Tornell, Enrique Hernández-Orallo, Carlos T. Calafate, Juan-Carlos Cano and Pietro Manzoni \\ Universitat Politècnica de València \\ Camino de Vera, s/n, 46022 Valencia, Spain \\ sermarto@upv.es, \{ehernandez, calafate, jucano,pmanzoni\}@disca.upv.es
}

\begin{abstract}
The Delay Tolerant Networks (DTN) approach is considered the best strategy to address the specific issues of the VANETs, namely high mobility, variable node density or frequent radio obstacles. Several protocols have been proposed for DTNs, being the epidemic routing (and variations of it) the most representative protocol. Nevertheless, the availability of navigation systems, thanks to which each vehicle is aware of its location within a map, introduces the possibility for a new routing approach, known as Geographic Routing.

In this paper we analytically evaluate the performance of our previously presented Map-based Sensor-data Delivery Protocol (MSDP). We introduce an analytical model that takes into account the effect of constrained buffers. The results show that adopting the Map-based Sensor-data Delivery Protocol (MSDP) routing mechanism allows achieving a reasonable delivery time with an insignificant overhead compared with epidemic routing.
\end{abstract}

Index Terms-Wireless Networks, DTNs, VANETs, Wireless sensor networks, GPS.

\section{INTRODUCTION}

Vehicular Ad-Hoc Networks (VANETs) turn every mobile car into a wireless router with forwarding capabilities. For the automotive industry, VANETs are proposed to improve safety-related and data communication among vehicles and between vehicles and Road Side Units (RSUs). Regarding safety-related communications, vehicles have different sensors which collect information not only about engine status, or speed, but also context information (e.g. weather or traffic status). This information must be collected and sent to data centers using VANET technologies as a way to improve road security and traffic management.

VANETs present some specific characteristics, like high speed and the presence of obstacles, like buildings, that produce a high variability in the network topology. Under these conditions we propose the use of Delay Tolerant Networks (DTNs) to deliver data obtained from the vehicles to an RSU. DTNs allow sharing information even in the presence of high delays. In DTNs, when a route to the destination of a message does not exists, the message is stored and carried until a route becomes available (this is known as the "store-carry-forward" paradigm).

Several protocols have been proposed for DTNs. The Epidemic Protocol [1] is based on copying the sender packet to a new node when a contact occurs (it infects the node) until one of the infected nodes contacts the destination node. This approach leads to a waste of resources when the number of nodes increases. To reduce the amount of generated network traffic some modifications have been proposed( [2], [3], [4], [5], etc) however none of them was specifically designed for VANETs.

A common assumption of VANET protocols is the availability of a Navigation System at the nodes. This way, each vehicle is aware of its geographical location. This information can be used to increase the efficiency of packet delivery in DTNs. This introduces a second group of routing algorithms usually called Geographic Routing. Some authors have focused on the direction of nodes as a decision parameter [6], obviating that in VANETs node mobility is constrained by street topology, and so the direction of the nodes, especially in urban environments, is rarely going to be constant. In this context, GeOpps [7] uses the information obtained from the Navigation System (NS) to determine the closest point to the destination along the route of a node. The next forwarding node is the one whose route passes closer or arrives sooner to the destination; this proposal has some problems that have been partially solved in GeoDTN+NAV [8]. GeoDTN+NAV adds a preceding step: before starting the DTN routing, it tries to find a path using GPSR [9] in order to reduce the delay. GeoSpray [10] is another protocol closely related with GeOpps that uses a multicopy scheme.

In a previous paper we introduced the Map-based Sensordata Delivery Protocol (MSDP) [11], a DTN routing protocol that, using the information obtained from a Geographic Information Service and the real street/road layout obtained from a Navigation System, attempts to find the best next forwarding node.

In this paper we evaluate the performance of MSDP using analytical models. We compare our protocol against the Epidemic routing protocol since the latter achieves an optimal delivery time when there are no buffer restrictions or congestion issues [12]; however, it introduces a great overhead due to the high number of messages transmitted. Since mobile nodes have limited storage capacity and work under congestion conditions, we also compare MSDP with the more realistic restrained Epidemic routing protocol.

The results of the analytical model show that MSDP routing has a reasonable delivery time with a insignificant overhead compared with the unrestricted epidemic routing. However, if we compare the results with the buffer-restricted epidemic routing, we can observe that, as we increase the load in the 
network, the delivery time also increases, reaching higher values than those obtained by the MSDP approach. Furthermore, under MSDP routing, the load has no effect on the delivery time or the overhead.

\section{MSDP OVERVIEW}

The goal of MSDP [11] is to efficiently transmit the information gathered from vehicular sensor networks to the RSUs. The RSUs locations have been chosen by an involved entity, and are connected to a traffic control center using a backbone network. Two networks interfaces are involved in this information delivery: an IEEE 802.11p interface for Carto-Car (C2C) communication, and an IEEE 802.11n interface for Car-to-Infraestructure (C2I) communication (that is, between vehicles and RSUs). In addition, it is mandatory that all mobile nodes (vehicle) have a certain degree of knowledge about their own route retrieved from a Navigation System (NS). The NS is defined as an interface which provides some minimal services, and it may simply be a preloaded static route plus a location service.

The basis of the MSDP protocol is a defined UtilityIndex (UI). This index is used to make the routing decisions and it is based on several factors, such as the trustworthiness of the NS, the time to reach an RSU, and the transmission availability. In other words, a higher UI value indicates that the node is a better candidate to reach the RSU. The UI is defined by the following function:

$$
\mathrm{UI}=\frac{P^{2}}{T} * Q
$$

Where the three parameters are defined as follows: trustworthiness factor $P$, quantifies the reliability of the information obtained from the NS related with the future route of the node; time to reach an RSU T; and transmission availability $Q$, that is the average transmission rate of each RSU through the NS. They are fully described in our previous work [11].

\section{AnALYTICAL MODELing}

In this section we model the performance of the MSDP and the Epidemic routing using Markov chains. The goal is to obtain the time that a packet needs to be delivered to the destination nodes (that is, the RSU nodes) and the cost (the number of hops or transmitted messages). Using this model we can compare our MSDP scheme with the Epidemic routing approach. The Epidemic routing is optimal in delivery time, but assumes that all nodes have sufficient space to store all packets. However, mobile nodes have limited storage capacity, so we also compare the MSDP routing with the more realistic constrained buffer Epidemic routing (we call it, the restricted epidemic routing).

For our models we assume that the rate of contacts between two mobile nodes and a mobile node or a static node (that is, the RSU node) follows an exponential distribution. Recent works show that the occurrence of contacts between two mobile nodes follows an exponential distribution with rate $\lambda$ [13] [16]. This has been shown valid specially for VANETs, considering vehicle-to-vehicle communications as well as with the roadside infrastructure (vehicle-to-roadside communications) [14]. There is some controversy about whether or not this exponential distribution can reflect some real mobility patterns. Empirical results have shown that the aggregated inter-contact times distribution follows a power-law and has a long tail [17]. In [18] it is shown that, in a bounded domain (such as the one selected along this paper), the inter-contact distribution is exponential but in an unbounded domain, it follows a powerlaw distribution instead. Therefore, we consider that using an exponential fit is a good choice to model inter-contact times. Moreover, by using exponential distributions we can formulate analytical models using Markov chains.

The network is modeled as a set of $M$ wireless mobile nodes and $R$ fixed destination nodes (RSU nodes). There are two vehicles contact rates: $\lambda_{M}$ is the mean contact rate between mobile nodes (that is, inside the set of M nodes) and $\lambda_{R}$ is the mean contact rate between mobile nodes and RSU nodes (that is, between the two sets). Upon contact, the packet can be transmitted. Nevertheless, a contact does not always imply a transmission. There are several factors that can reduce this transmission, for example the contact duration is too short to transmit the packet, other packets are transmitted before, or error transmissions occur. Thus, we introduce two new parameters into the model: the probability that a packet is successfully transmitted (or forwarded) between mobile nodes $\left(p_{t M}\right)$, and the probability of transmission between a mobile node and the RSU nodes $\left(p_{t R}\right)$.

\section{A. Modeling Epidemic diffusion}

In this section we derive a model for evaluating the time and cost of reaching the destination node for epidemic routing. First, we introduce a model for unrestricted epidemic diffusion (there is no buffer limitation in the nodes), and then we introduce a model for constrained buffer epidemic diffusion.

Several models has been proposed to evaluate the performance of Epidemic routing. Markov chain models were introduced in [13] for epidemic routing and 2-hop forwarding, deriving the average source-to-destination delivery delay and the number of existing copies of a packet at the time of delivery. The model in [12], which is based on Ordinary Differential Equations (ODE), obtained similar results. The previous models assume that all nodes are mobile with a unique contact rate and full probability of transmission when a contact occurs $\left(p_{t M}=1\right)$. Thus, we extend the Markov Chain model to include the mobile and destination set of nodes with their different contact rates $\left(\lambda_{M}\right.$ and $\left.\lambda_{R}\right)$ and the probabilities of transmission $\left(p_{t M}\right.$ and $\left.p_{t R}\right)$.

The basis of the model is a 2D Continuous Time Markov chain (2D-CTMC) with states $(d(t), m(t))_{t \geq 0}$, where $m(t)$ (and $d(t)$ ) represents the number of mobile (and destination) nodes that have the packet at time $t$. At the beginning only one mobile node (the sender node) has the packet. Then, when a mobile contact occurs, $m$ can be increased by one with probability $p_{t M}$. Alternatively, when a mobile contacts with a destination node (with rate $\lambda_{R}$ ), $d$ can be increased by one with probability $p_{t R}$. The final absorbing states are when 
$d>0$. Thus, this 2D-CTMC has an initial state $s_{1}=(0,1), M$ transient states (from $s_{1}=(0,1)$ to $s_{\tau}=(0, M)$ states) and $M$ absorbing states (from $s_{\tau+1}=(1,1)$ to $s_{\tau+v}=(1, M)$ ). We define $\tau$ as the number of transient states $(\tau=M)$ and $v$ as the number of absorbing states $(v=M)$. This model can be expressed using the following transition matrix $\mathbf{P}$ in the canonical form:

$$
\mathbf{P}=\left(\begin{array}{cc}
\mathbf{Q} & \mathbf{R} \\
\mathbf{0} & \mathbf{I}
\end{array}\right)
$$

where $\mathbf{I}$ is a $v \times v$ identity matrix, $\mathbf{0}$ is a $v \times \tau$ zero matrix, $\mathbf{Q}$ is a $\tau \times \tau$ matrix with elements $p_{i j}$ denoting the transition rate from transient state $s_{i}$ to transient state $s_{j}$ and $\mathbf{R}$ is a $\tau \times v$ matrix with elements $p_{i j}$ denoting the transition rate from transient state $s_{i}$ to the absorbing state $s_{j}$.

Now, we derive the transition rates $p_{i j}$. Given the state $s_{i}=$ $(d, m)^{1}$ the following transitions can occur:

- $(d, m)$ to $(d, m+1)$ : A new mobile node has the packet, due to a contact between mobiles nodes with rate $\lambda_{M}$. Thus, the transition probability is $t_{m}=\lambda_{M} p_{t M} \cdot m(M-$ $m$ ) where $(M-m)$ represents the number of pending mobiles nodes that can receive the packet.

- $(0, m)$ to $(1, m)$ : An RSU node has the packet, due a contact between a mobile node and a destination node with rate $\lambda_{R}$. Thus, the transition probability is $t_{r}=$ $\lambda_{R} p_{t R} \cdot m R$.

- $(d, p)$ to $(d, p)$ : This is the probability of no changes and is $1-\sum_{j \neq i} p_{i j}$.

Using the transition matrix $\mathbf{P}$ we can derive the delivery time $T_{d}$. From the 2D-CTMC we can obtain how long will it take for the process to be absorbed. Using the fundamental matrix $\mathbf{N}=(\mathbf{I}-\mathbf{Q})^{-1}$, we can obtain a vector $\mathbf{t}$ of the expected time to absorption as $\mathbf{t}=\mathbf{N v}$, where $\mathbf{v}$ is a column vector of ones $\left(\mathbf{v}=[1,1, \ldots, 1]^{T}\right)$. Each entry $t_{i}$ of $\mathbf{t}$ represents the expected time to absorption from state $s_{i}$. Since we only need the expected time from state $s_{1}=(0,1)$ to absorption, the delivery time $T_{d}$, is:

$$
T_{d}=E[T]=\mathbf{v}_{\mathbf{1}} \mathbf{N v}
$$

where $T$ is a random variable denoting the delivery time for all nodes and $\mathbf{v}_{\mathbf{1}}=[1,0, \ldots, 0]$.

Now, we calculate the overhead, that is, the mean number of copies (or replicas) of the packet until the delivery time. If we assume that a packet is not transmitted again to a node that already has it, the number of copies is equivalent to the number of transmissions. Therefore, the number of copies is done calculating the average number of packets transmitted in each state $s_{i}$. To do this, we obtain the duration of each state $s_{i}$ using the fundamental matrix N. By definition, the elements of the first row of $\mathbf{N}$ are the expected times in each state starting from state 0 . Then, the duration of state $s_{i}$ is $\mathbf{N}(1, i)$. In state $s_{1}=(0,1)$ only one node has the packet, and this packet can be transmitted to all nodes (except himself), that is $M-1$ nodes, for the duration of this state (denoted as $\mathbf{N}(1,1)$ )

\footnotetext{
${ }^{1}$ For simplicity, we omit the time in the states (that is $(d, m)=$ $(d(t), m(t))$
}

with a rate $\lambda_{M}$ and probability $p_{c}$. Then for state $s_{2}=(0,2)$ two nodes have the packet and it can be transmitted to $M-2$ nodes. Thus, for state $s_{i}=(0, m), i \leq \tau$, the average number of copies in this state is $\lambda_{M} p_{t M} \mathbf{N}(1, i) m(M-m)$. Summing up, the overhead (or the expected number of copies) is:

$$
O_{d}=E[C]=\lambda_{M} p_{t M} \sum_{m=1}^{\tau} \mathbf{N}(1, i) m(M-m)
$$

Note that previous expressions for time and copies obtain the same results than equations in [12] when $\lambda_{M}=\lambda_{R}, R=1$ and $p_{t M}=p_{t R}=1$, that is, $T_{d}=\frac{\log M}{\lambda(M-1)}$ and $O_{d}=\frac{M-1}{2}$.

\section{B. Constrained buffer}

In the unrestricted epidemic routing there are no constraints on the number of packet replicas in the network. Now we derive a model for Epidemic routing under constrained buffer (the restricted epidemic routing). In this case, we assume that mobile nodes have a limited buffer of size $B$ (that is, they can only store $B$ packets). For the destination nodes, we keep the assumption of unrestricted buffer size (they are fixed nodes, so memory is not a problem).

First, we need to obtain the average buffer occupancy. We consider the approximation derived in [12] for the case of $F$ unicast flows. Each flow generates packets following a Poisson process with rate $\delta$. Then, the average queue size is:

$$
E[Q]=\frac{F \delta}{M \lambda_{M}} 2 E[C]
$$

Using this expression we simply define a new probability of transmission $P_{t}$ that will depend on the average buffer occupancy. That is, if the buffer is full then we can transmit the packet if another one is dropped from the buffer. Assuming a random dropping, we have the following probability of transmission:

$$
P_{t}= \begin{cases}p_{t M} & E[Q]<B \\ \frac{B}{E[Q]+1} p_{t M} & E[Q] \geq B\end{cases}
$$

This value is used for calculating the transition probability of $(d, m)$ to $(d, m+1), t_{m}=\lambda_{M} P_{t} \cdot m(M-m)$. Using this new transition probability we can obtain the time and overhead using equations 3 and 4 . Note, that in order to obtain $E[Q]$ we need a prior value of $E[C]$, that is one of the results of the model. So this value is iteratively approximated from an initial value $E[C]^{0}$ obtained with the unrestricted epidemic model, and then calculating values of $E[C]^{x+1}$ using the restricted epidemic model with $E[C]^{x}$ until a given convergence criteria is reached (that is, the difference between the successive values is less than a given error $\epsilon$ )

\section{Modeling MSDP}

Now, we are going to model our MSDP protocol. Without loss of generality we focus our study to only one destination node $(R=1)$. In the MSDP protocol there is only one packet in the network that is stored in the custodian node. When a contact occurs the packet is transmitted to a new node if the UtiliyIndex (UI) of the receiver (candidate) node is greater 


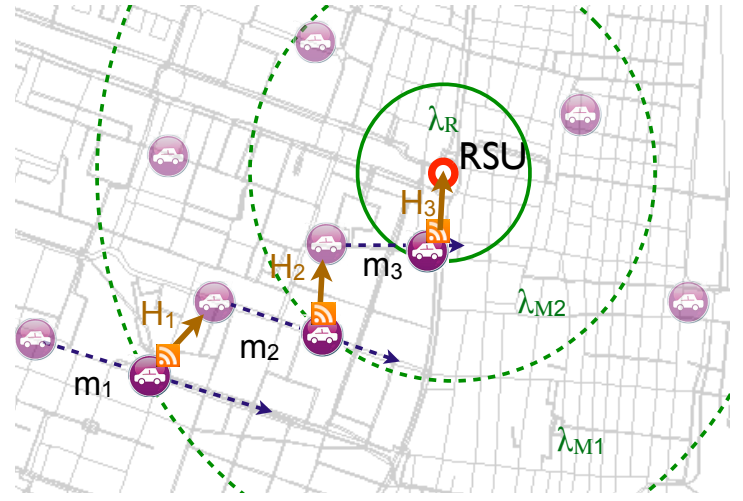

Fig. 1: Typical situation for MSDP, dashed lines represent the movement of the nodes, while solid lines represent wireless transmissions.

than the UI of the sender node. This way the UI reflects how near is a node to the destination RSU node. Basically, the higher the UI, the nearer to the RSU node. Figure 1 shows an example of packet delivery. It starts with $m_{1}$ as the sender node. When a contact occurs with $m_{2}$, this node has an UI greater than $m_{1}$ so the packet is transmitted (first hop, $H_{1}$ ). For the following hop $\left(\mathrm{H}_{2}\right)$, the UI of the node that has the packet is increased. Finally, the packet reaches the destination node. The UI has another property, the locality. Two nodes with similar UI values are prone to be neighbors. So it is more frequent that a contact occurs between these nodes and other nodes in their neighborhood (that is, they have a greater contact rate). Following the example of figure 1, when the packet is in $m_{1}$, the contact rate with all the nodes inside the circumference defined by the boundary of the node is $\lambda_{M 1}$. When the packet is transmitted to $m_{2}$, the circumference is reduced and the contact rate is increased $\lambda_{M 2} \geq \lambda_{M 1}$. Therefore, we expect a direct relation between the UI of a node and the contact rate.

This is confirmed with the following experiment. From the simulation scenario we used in our previous paper [11], we obtained all the inter-contact times between nodes and the UI of the sender node when a contact occurs. If we sort the nodes by increasing UI values, the UI position is the index on this list. Figure 2 shows the plot of the contact rate depending on the UI position for $M$ nodes. We can clearly observe that the contact rate increases with the UI position. That is, if we have $M$ mobile nodes, the list is: $\left\{\mathrm{UI}_{1}, \mathrm{UI}_{2}, \ldots \mathrm{UI}_{i}, \mathrm{UI}_{j}, \ldots \mathrm{UI}_{M}\right\}$, so $\mathrm{UI}_{j} \geq \mathrm{UI}_{i} \forall j>i$. This list is dynamic, so a node can change its position over time. The probability of changing one position is defined as $p_{u}$. As we sort nodes by their UI values, we can establish a direct relation between the UI position and the contact rate. Thus, we can fit a third degree polynomial function $f_{\lambda}(i, j)$, that gives the contact rate of two nodes with position index $i$ and $j$ :

$$
f_{\lambda}(i, j) \approx c_{4}+c_{3} k+c_{2} k^{2}+c_{1} k^{3} \quad k=\min (i, j) \quad i \neq j
$$

Note that $k=\min (i, j)$ reflects the fact that the contact rate for two nodes is determined by the lowest index. In the example of figure 2, the contact rate between nodes $m_{1}$ and $m_{3}$ is $\lambda_{M 1}$. Finally, figure 2 shows the result of fitting this

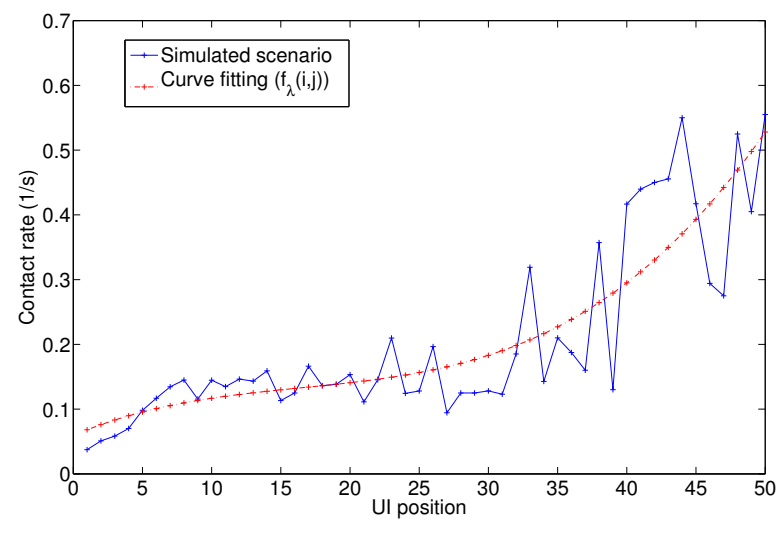

Fig. 2: Contact rate depending on the UtilityIndex.

curve to the values obtained from the scenario. We can see the effect of the logarithm effect in the calculus of the time to reach factor $(T)$ of the UI expression, specially for UI positions greater than 30 .

The contact rate for the destination nodes (RSU nodes) follows a similar distribution, so the higher the index $i$ of a node the higher the contact rate, and we can also fit a similar equation $f_{\lambda}^{\prime}(i)$.

Using a CMTC we can obtain the time to reach the destination and the overhead (in this case, the number of hops until the packet arrives to any of the RSU nodes). We introduce $H$ as the maximum number of possible hops $(H \leq M)$. Following the same process that in the epidemic model, we have a 3D-CMTC with states $(d(t), u(t), h(t))_{t \geq 0}$ where $h(t)$ is the number of hops at time $t, u(t)$ is the position on the list of UI at time $t$ and $d(t)$ represents if the destination node have the packet at time $t$. At the beginning we start with $h=0$ hops, but we assume that the sender can be any node of the mobile nodes so its average index position $u$ is in the middle: $\lfloor M / 2\rfloor$. Therefore, the starting state is $s_{\alpha}=(0,\lfloor M / 2\rfloor, 0)^{2}$. The final (absorbing) states is when $d=1$. Thus, this 3DCTMC has $M(H+1)$ transient states (from $s_{1}=(0,1,0)$ to $s_{\tau}=(0, M, H)$ states) and $M(H+1)$ absorbing states (from $s_{\tau+1}=(1,1,0)$ to $\left.s_{\tau+v}=(1, M, H)\right)$.

Now, we derive the transition rates $p_{i j}$. Given the state $s_{i}=$ $(d, m, h)$ the following transitions can occur:

- $(d, u, h)$ to $(d, u+\Delta, h+1), \Delta=1 \ldots(M-u)$ : The packet is transmitted to a new node with a greater UI. The contact rate depends on the difference of the UI of the nodes contacted: $f_{\lambda}(i, j)$. Thus, the transition probability is $t_{u h}=f_{\lambda}(i, j) \cdot p_{t M}$.

- $(d, u, h)$ to $(d, u \pm 1, h)$ : This transition reflects that the node that has the packet increases (decreases) one position in the UI list. The transition probability is simply $p_{u}$.

- $(0, u, h)$ to $(1, u, h+1)$ : An RSU node has the packet. The contact rate depends on the value of $u: f_{\lambda}^{\prime}(u)$. Thus, the transition probability is $t_{d}=f_{\lambda}^{\prime}(u) \cdot p_{t R}$.

${ }^{2}$ We can convert from an state $(d, u, h)$ to a state number $i$ using the following expression: $i=\operatorname{State}(d, u, h)=d \cdot M(H+1)+u(H+1)+h+1$, so the starting state number $\alpha$ is $\lfloor M / 2\rfloor(H+1)$ 
- $(d, u, h)$ to $(d, u, h)$ : This is the probability of no changes and is $1-\sum_{j \neq i} p_{i j}$.

Using the transition matrix $\mathbf{P}$ we derive the delivery time $T_{d}$ using an expression similar to equation 3 :

$$
T_{d}=E[T]=\mathbf{v}_{\alpha} \mathbf{N v}
$$

where $\mathbf{v}_{\alpha}$ is a vector with a 1 in the start state $\alpha$.

Now, we derive the overhead (the number of hops or retransmissions). First, we obtain the matrix of absorption probabilities as $\mathbf{B}=\mathbf{N} \cdot \mathbf{R}$. Then, we obtain the probability of absorption $\left(p_{H}\right)$ depending on the number of hops starting from state number $\alpha$ :

$$
p_{H}(h)=\sum_{u=1}^{M} B(\alpha, \operatorname{State}(0, u, h)) \quad h=1 \ldots H
$$

Thus, $p_{H}$ is the probability mass function $(p m f)$, that gives the probability of absorption (that is, the packet reaches the destination) with $h$ hops. Using this pmf we can obtain the cumulative distribution functions $F_{H}(h)$. Then, the average number of hops needed to reach the RSU node $E[H]$ is the greater value of $h$ that make true the expression $F_{H}(h) \leq 0.5$. That is:

$$
O_{d}=E[H]=\max \left\{h \mid F_{H}(h) \leq 0.5\right\}
$$

As in the Epidemic model, we can also consider the effect of the buffer, although in the MSPD its influence will be limited. Assuming the same $F$ unicast flows, the arrival rate of new packet to the network is $F \delta$, and by Little's law, the average number of packets in the system is $F \delta E[T]$, where $E[T]=T_{d}$ is precisely the average packet lifetime. If all these packets are equally divided among the $M$ nodes we have that the average queue size is:

$$
E[Q]=\frac{F \delta}{M} E[T]
$$

Using this average queue size we can obtain the probability of transmission $P_{t}$ using equation 6 in order to calculate the new transition probability $t_{u h}=f_{\lambda}(i, j) \cdot P_{t}$.

\section{ANALYTICAL PERFORMANCE EVALUATION}

In this section we make an analytical comparison of the performance of the MSDP protocol with the epidemic routing approaches using the previously presented models. In this evaluation we use the following parameters that were derived from the simulation scenario we used in [11]: $\lambda_{M}=0.141$, $\lambda_{R}=0.046, p_{t M}=0.5, p_{t R}=0.7, p_{U}=0.05, H=20$, $R=1$. The coefficients of the $f_{\lambda}$ functions were obtained through a curve fit based on the simulation results, as shown previously. Figure 3 presents the time and overhead depending on the number of mobile nodes. In figure $3 \mathrm{a}$ we can see the delivery time. Regarding the unrestricted protocols, results show that for MSDP the delivery time is about ten times greater than for Epidemic. Note that the epidemic routing is optimal in delivery time, but has a great overhead, as we can see in figure $3 \mathrm{~b}$. The average number of transmissions for the

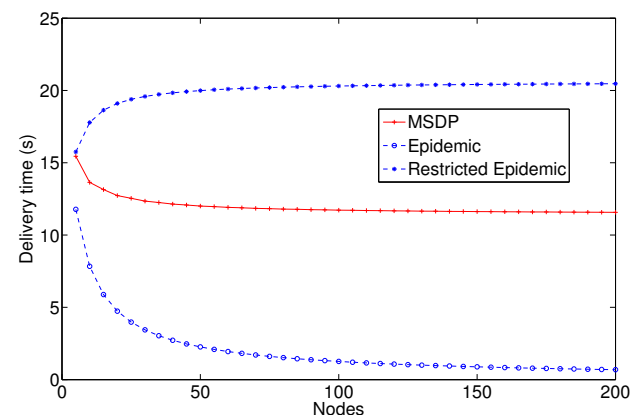

(a)

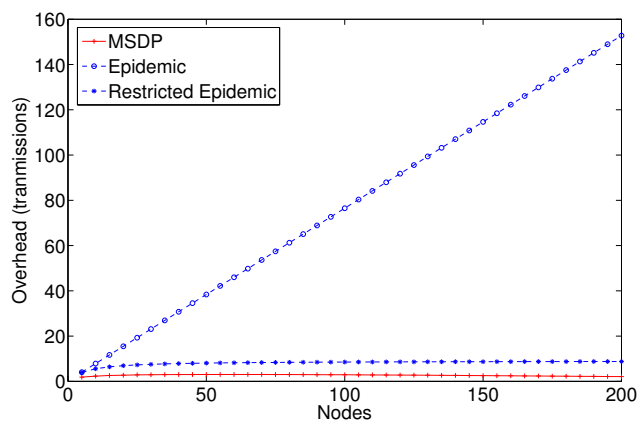

(b)

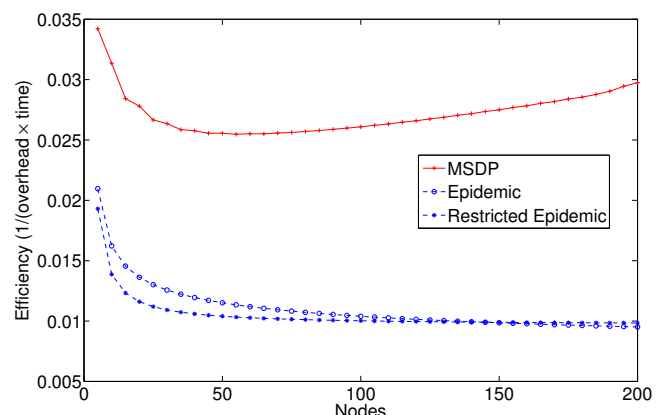

(c)

Fig. 3: Evaluation of the MSDP protocol with the Epidemic protocols a) Delivery time; b) Overhead: average number of transmitted packets; c) Efficiency of the protocols.

Epidemic protocols increases linearly with M, while for MSDP it increases more slowly.

The results for buffer restricted epidemic protocols are totally different. We used the following values: a buffer of 50 packets $(B=50)$ and all nodes send a message to the RSU $(F=M)$ every five seconds. This message is fragmented in ten packets so $\delta=2$. Note that MSDP has only one copy of each packet in the network, and so this does not imply an increase on network load; also, the effect of the buffer restriction in this evaluation is negligible. Regarding the overhead, we also see that the number of copies is reduced when the load increases. Finally, in figure $3 \mathrm{c}$ we can see the efficiency of the protocols obtained as $\left(O_{d} \times T_{d}\right)^{-1}$, so a higher value implies a more efficient protocol. Thus, MSDP is about 10-20 times more efficient than the epidemic protocols.

Figure 4 shows the delivery time depending on the number of flows $(F)$ in a network with 100 mobile nodes $(M=100)$ using the same parameters of previous experiments. We can see an exponential growth of the time for low values of $F$. The 


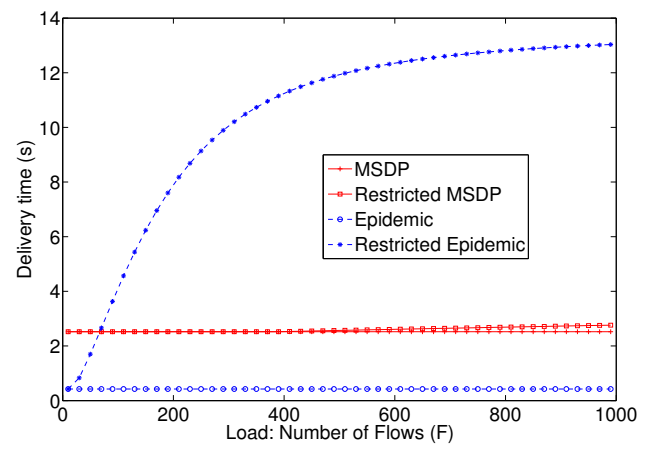

Fig. 4: Delivery time depending on load ( $F=$ number of flows)

limit is reached when the network buffers are saturated, so the delivery is made through a direct contact between the sender and the receiver. For restricted MSDP, as only one copy of each packet is present in the network, he effect is quite reduced, as we can appreciate it in the same figure. The increase on the delivery time is minimal (about $5 \%$ for 1000 flows of load).

The previous evaluations show that the effect of network load has low influence on the efficiency of the MSDP protocol, allowing to obtain good delivery times in a very efficient way. This analytical results confirm the results we obtained trough simulations in [11].

\section{CONCLUSIONS AND FUTURE WORK}

This paper evaluates the performance of our proposed Map-based Sensor-data Delivery Protocol (MSDP). MSDP is a DTN geographic routing protocol that uses the locations and routes of vehicles, coupled with information obtained from the Navigation System (NS), in order to determine the best forwarding node. Moreover, the protocol considers other parameters such as the buffer load or the trustworthiness of the node. Therefore, when a packet is transmitted to a new node, its probability of reaching the destination node increases. As long as there is only one packet in the network, the overhead incurred is low in comparison to multi-copy schemes.

To evaluate our protocol we have compared it with the Epidemic routing protocol. We introduced analytical models of the epidemic and MSDP protocols. These models take into account the buffer constraints, whose effects are especially important when evaluating the performance of epidemic routing.

The evaluations showed that MSDP has a reasonable delivery time with a reduced overhead compared with the epidemic routing solutions evaluated. Considering real network restrictions (buffer and congestion), the MSDP delivery time is lower than the epidemic routing, while behaving more efficiently. This is due to the low channel usage (that is, overhead) associated to our MSDP approach, which avoids the congestion effects of epidemic routing.

\section{ACKNOWLEDGMENTS}

This work was partially supported by the Ministerio de Ciencia e Innovación, Spain, under Grants TIN2011-27543C03-01 and BES-2012-052673.

\section{REFERENCES}

[1] A. Vahdat and D. Becker, "Epidemic Routing for Partially Connected Ad Hoc Networks," in Technical Report CS-200006, Apr. 2000.

[2] T. Spyropoulos, K. Psounis, and C. S. Raghavendra, "Spray and wait: an efficient routing scheme for intermittently connected mobile networks," in Proceedings of the 2005 ACM SIGCOMM workshop on Delaytolerant networking, ser. WDTN '05. New York, NY, USA: ACM, 2005, pp. 252-259.

[3] J. Xue, X. Fan, Y. Cao, J. Fang, and J. Li, "Spray and Wait Routing Based on Average Delivery Probability in Delay Tolerant Network," in Networks Security, Wireless Communications and Trusted Computing, 2009. NSWCTC '09. International Conference on, vol. 2. IEEE, Apr. 2009, pp. 500-502.

[4] A. Lindgren, A. Doria, and O. Schelén, "Probabilistic routing in intermittently connected networks," in SIGMOBILE Mob. Comput. Commun. Rev., vol. 7. New York, NY, USA: ACM, Jul. 2003, pp. 19-20.

[5] T.-K. Huang, C.-K. Lee, and L.-J. Chen, "PRoPHET+: An Adaptive PRoPHET-Based Routing Protocol for Opportunistic Network," in Advanced Information Networking and Applications (AINA), 2010 24th IEEE International Conference on. IEEE, Apr. 2010, pp. 112-119.

[6] Z. Li and H. Shen, "A Direction Based Geographic Routing Scheme for Intermittently Connected Mobile Networks," in Embedded and Ubiquitous Computing, 2008. EUC '08. IEEE/IFIP International Conference on, vol. 1. IEEE, Dec. 2008, pp. 359-365.

[7] I. Leontiadis and C. Mascolo, "GeOpps: Geographical Opportunistic Routing for Vehicular Networks," in World of Wireless, Mobile and Multimedia Networks, 2007. WoWMoM 2007. IEEE International Symposium on a. IEEE, Jun. 2007, pp. 1-6. [Online]. Available: http://dx.doi.org/10.1109/WOWMOM.2007.4351688

[8] P. C. Cheng, K. C. Lee, M. Gerla, and J. Härri, "GeoDTN+Nav: Geographic DTN Routing with Navigator Prediction for Urban Vehicular Environments," Mob. Netw. Appl., vol. 15, pp. 61-82, Feb. 2010. [Online]. Available: http://dx.doi.org/10.1007/s11036-009-0181-6

[9] B. Karp and H. T. Kung, "GPSR: greedy perimeter stateless routing for wireless networks," in Proceedings of the 6th annual international conference on Mobile computing and networking, ser. MobiCom '00. New York, NY, USA: ACM, 2000, pp. 243-254.

[10] V. N. G. J. Soares, J. J. P. C. Rodrigues, and F. Farahmand, "GeoSpray: A Geographic Routing Protocol for Vehicular DelayTolerant Networks," Information Fusion, Nov. 2011. [Online]. Available: http://dx.doi.org/10.1016/j.inffus.2011.11.003

[11] S. M. Tornell, C. T. Calafate, J.-C. Cano, and P. Manzoni, "A Map-Based sensor data delivery protocol for vehicular networks," in 2012 The 11th Annual Mediterranean Ad Hoc Networking Workshop (Med-Hoc-Net) (Med-Hoc-Net'12), Ayia Napa, Cyprus, Jun. 2012.

[12] X. Zhang, G. Neglia, J. Kurose, and D. Towsley, "Performance modeling of epidemic routing," Computer Networks, vol. 51, no. 10, pp. 2867 2891, 2007.

[13] R. Groenevelt, P. Nain, and G. Koole, "The message delay in mobile ad hoc networks," Performance Evaluation, vol. 62, pp. 210-228, October 2005.

[14] H. Zhu, L. Fu, G. Xue, Y. Zhu, M. Li, and L. M. Ni, "Recognizing exponential inter-contact time in vanets," in Proceedings of the 29th conference on Information communications, ser. INFOCOM'10. Piscataway, NJ, USA: IEEE Press, 2010, pp. 101-105.

[15] Y. Li, G. Su, D. Wu, D. Jin, L. Su, and L. Zeng, "The impact of node selfishness on multicasting in delay tolerant networks," Vehicular Technology, IEEE Transactions on, vol. 60, no. 5, pp. $2224-2238$, jun 2011.

[16] W. Gao, Q. Li, B. Zhao, and G. Cao, "Multicasting in delay tolerant networks: a social network perspective," in Proceedings of the tenth ACM international symposium on Mobile ad hoc networking and computing, ser. MobiHoc '09. New York, NY, USA: ACM, 2009, pp. 299-308.

[17] A. Chaintreau, P. Hui, J. Crowcroft, C. Diot, R. Gass, and J. Scott, "Impact of human mobility on opportunistic forwarding algorithms," IEEE Transactions on Mobile Computing, vol. 6, pp. 606-620, June 2007.

[18] H. Cai and D. Y. Eun, "Crossing over the bounded domain: From exponential to power-law intermeeting time in mobile ad hoc networks," Networking, IEEE/ACM Transactions on, vol. 17, no. 5, pp. $1578-1591$, oct. 2009 . 